\title{
Reversible Nail Discoloration from Hydroquinone 4\% Cream
}

\begin{abstract}
Introduction: Hydroquinone is a tyrosinase-inhibiting phenolic compound widely prescribed for disorders of pigmentation such as melasma, lentigines and post inflammatory hyper pigmentation. Well-known adverse effects of topical hydroquinone include allergic contact dermatitis and exogenous ochronosis after long-term use. Pigmentation of the nails is a nother rela tively unc ommon yet reversible treatment complication.

Observation: A 38-year-old female presented with hyperpigmentation of her fingernails after daily application of $4 \%$ hydroquinone to the face for 3 months. Physical examination revealed brownish, ill-defined, macular discoloration $4-6 \mathrm{~mm}$ distal to the proximal nail fold and the fourth and fifth fingernail beds of both hands but with more pronounced involvement of the right fingernails. Examination of the palms showed brownish discoloration of calluses overlying the heads of the metacarpals. The brown discoloration of the fingernails could not be scrapped off with a 15-blade. Nail clippings sent for histopathology were notable for amorphous yellowbrown globules with Fontana-Masson staining. The nail pigmentation ultimately resolved 2 months after cessation of the hydroquinone product.

Conclusion: Hydroquinone-containing creams are widely available over the counter, and hydroquinone is generally considered safe and effective at concentrations of $4 \%$ or less. Although uncommon and self-limited, nail hyperpigmentation after topical hydroquinone is a possible side effect that dermatologists should be aware of. Considering the increasing use of hydroquinone-containing products for aesthetic purposes in the cosmetic area, not only dematologists but also other cosmetic practitioners should be familiar with this potential complication to better inform their patients.
\end{abstract}

\section{Introduction}

Hydroquinone remains the gold standard for hyperpigmentation therapy in the United Sates and is widely prescribed for disorders of pigmentation such as melasma, lentigines and post inflammatory hyperpigmentation [1]. This tyrosinase-inhibiting phenolic compound has a few well-known adverse effect such as allergic contact dermatitis and exogenous ochronosis after long-term use. However, pigmentation of the nails as a complication of topical hydroquinone is relatively uncommon, with only a few reports in the literature [26]. We present a case of nail hyperpigmentation after use of a $4 \%$ hydroquinone-containing cream for treatment of facial dyschromia.

\section{Case Report}

A 38-year-old female presented with hyperpigmentation of her fingernails after daily application of $4 \%$ hydroquinone to the face for 3 months. The patient was right-handed and reported no hand washing following product application.

Physical examination revealed brownish, ill-defined, macular discoloration 4-6 $\mathrm{mm}$ distal to the proximal nail fold involving the fourth and fifth fingernail beds of both hands but with more pronounced involvement of the right fingernails. The toenails were uninvolved. Examination of the palms showed brownish discoloration of calluses overlying the heads of the metacarpals. No other cutaneous and mucosal findings were noted. The brown discoloration of the fingernails could not be scrapped off with a 15-blade.Examination

\section{Journal of}

Clinical \& Investigative Dermatology

Thais Prota Hussein Pincelli*, Alison J. Bruce, Scott W. Fosko and Jason C. Sluzevich

Department of Dermatology, Mayo Clinic Florida, USA

\section{*Address for Correspondence}

Thais Prota Hussein Pincelli, 1400 W. Chinaberry Ct, St. Johns - FL 32259, USA, Tel: 904-584-5458; E-mail: pincelli.thais@mayo.edu

Submission: 28 February, 2018

Accepted: 12 March, 2018

Published: 19 March, 2018

Copyright: @ 2018 Pincelli TPH, et al. This is an open access article distributed under the Creative Commons Attribution License, which permits unrestricted use, distribution, and reproduction in any medium, provided the original work is properly cited.

of the lid of the hydroquinone product used by the patient showed a similar brown discoloration to that of her fingernails (Figures 1-4).

Nail clippings were obtained and sent for histopathology. GMS stain was negative. Amorphous yellow-brown globules were highlighted throughout the nail plate with Fontana-Masson staining (Figure 5). Perl's stain was unremarkable.

The nail pigmentation ultimately resolved 2 months after cessation of the hydroquinone product.

\section{Discussion}

Hydroquinone is a well-known inhibitor of melanin production that inhibits the enzymatic oxidation of tyrosine to dopa and its subsequent conversion to melanin. Fitzpatrick also proposed that hydroquinone inhibits not only the formation, melanization and degradation of melanosomes but also interacts with the membranous structures of melanocytes eventually causing necrosis of whole melanocytes [7].

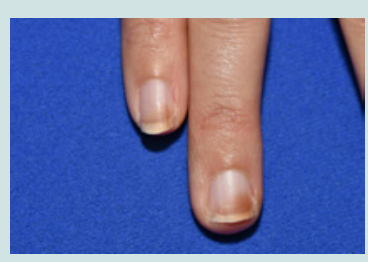

Figure 1: Brown discoloration of the distal aspect of the fourth and fifth fingernails at presentation.

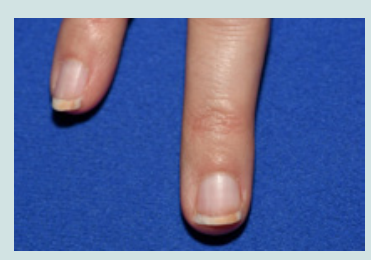

Figure 2: Resolution of pigmentation two months after cessation of hydroquinone product. 


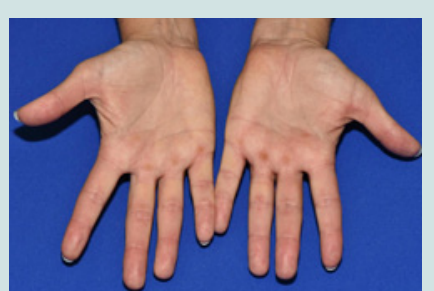

Figure 3: Brownish discoloration of calluses overlying the heads of the metacarpals on the palms.

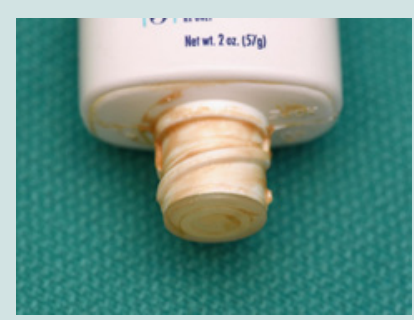

Figure 4: Brown discoloration on lid of hydroquinone product used by the patient.

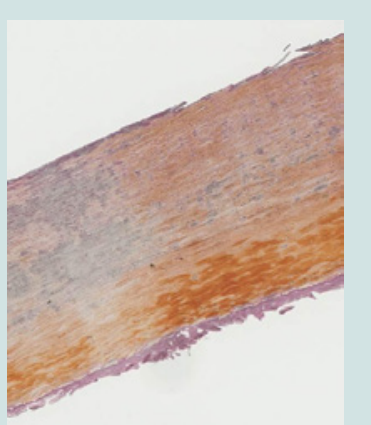

Figure 5: Histopathology with Fontana-Masson staining showed amorphous yellow-brown globules throughout the nail plate.

Only a few cases of nail staining by hydroquinone have been reported previously. All patients were Caucasian using 2-5\% hydroquinone topically [2-6].The acquired hyperpigmentation generally presented in the distal part of the fingernails of one or both hands, was asymptomatic, and worsened with sun exposure although this was not seen in this case. Cessation of the hydroquinonecontaining creams or use of gloves to protect the nails resulted in complete resolution of the discoloration within 1-3 months [2-6].
The nail pigmentation following use of topical hydroquinone may be the result of oxidation and polymerization products of hydroquinone. Hydroquinone undergoes oxidation to quinone, a yellow compound that is subsequently oxidized to hydroxyquinone in the presence of light. Hydroxyquinone is another yellow compound and further light exposure causes it to polymerize to products which are dark brown in color [2,3]. The resolution of the nail staining during the winter months in a patient reported by Mann et al strongly suggests that UV light may be an important etiologic cofactor [3]. The yellow-brown pigment histologically resembles the dermal deposits seen in exogenous ochronosis. This suggests hydroquinone and related degeneration products may chemically interact with epithelial keratins as well as dermal proteins.

Hydroquinone-containing creams are widely available over the counter, and hydroquinone is generally considered safe and effective at concentrations of $4 \%$ or less. Higher concentrations are associated with an increased risk of dermatitis and exogenous ochronosis. Although uncommon and self-limited, nail hyperpigmentation after topical hydroquinone is a possible side effect that dermatologists should be aware of. Patients prescribed topical hydroquinone should be warned to protect their nails by either wearing gloves before applying the product or by immediately washing their hands after the application. Considering the increasing use of hydroquinonecontaining products for aesthetic purposes in the cosmetic area, not only dermatologists but also other cosmetic practitioners should be familiar with this potential complication to better inform their patients.

\section{References}

1. Draelos ZD (2007) Skin lightening preparations and the hydroquinone controversy. Dermatol Ther 5: 308-313.

2. Parlak AH, Aydogan I, Kavak A (2003) Discolouration of fingernails from using hydroquinone skin lightening cream. J Cosmet Dermatol 2: 199-201.

3. Mann RJ, Harman RR (1983) Nail staining due to hydroquinone skin lightening creams. Br J Dermatol 108: 363-365.

4. Ozluer SM, Muir J (2000) Nail staining from hydroquinone cream. Australas J Dermatol 41: 255-256.

5. Garcia RL, White JW Jr, Willis WF (1978) Hydroquinone nail pigmentation. Arch Dermatol 14: 1402-1403.

6. Glazer A, Sofen BD, Gallo ES (2016) Nail discoloration after use of hydroquinone. JAAD Case Rep 1: 57-58.

7. Jimbow K, Obata H, Pathak MA, Fitzpatrick TB (1974) Mechanism of depigmentation by hydroquinone. J Invest Dermatol 62: 436-449.

8. Kramer KE, Lopez A, Stefanato CM, Phillips TJ (2000) Exogenous ochronosis J Am Acad Dermatol 42: 869-871. 